\title{
MAJOR COMPLICATIONS IN PATIENTS WITH INTRA-ABDOMINAL INFECTION. COULD PIRO, MANNHEIM PERITONITIS INDEX AND PERITONITIS SEPSIS SCORE BE USEFUL IN MORBIDITY PREDICTION?
}

Laura Castelltort, Laia Bosch, Adela Benítez-Cano, Marc Sadurní, Lluís Aguilera, Carlos García-Bernedo Hospital del Mar, Dept of Anaesthesiology \& Intensive Care, Barcelona, Spain.

\section{BACKGROUND AND GOAL OF THE STUDY}

PIRO (Predisposition, Insult/Infection, Response and Organ disfunction) score, Mannheim Peritonitis Index (MPI) and Peritonitis Sepsis Score (PSS) are useful scores to predict mortality in patients with intraabdominal infection (IAI). However, these scores do not estimate morbidity, clearly related with clincal and economic impact on septic patients admitted in a surgical intensive care unit (SICU). The goal of the study is to evaluate the prediction capacity of the PIRO, MPI and PSS scores in terms of cardiovascular, renal and respiratory morbidity.

\section{MATERIAL AND METHODS}

A retrospective observational study was performed (January 2015 to August 2017) in patients admitted to a tertiary SICU with IAI and intraoperative positive cultures. Demographic, clinical, microbiological and surgical variables were recorded to calculate PIRO score, MPI and PSS.

Cardiovascular morbidity include cardiac events (myocardial infarct and stroke) and vasoactive drug support longer than 24 hours. Respiratory complications were evaluated with invasive or noninvasive mechanical ventilation requirement. Postoperative renal support therapy was the renal complication evaluated.
The evaluation of prediction accuracy for each score was performed comparing, for each endpoint, ROC curves and its areas (AUC). The value of marker defined as cut-off was determined by the maximum of Youden index.

\section{RESULTS AND DISCUSSION}

A total of 129 patients were analyzed with a mean (SD) age of 62 years $(17,4), 61 \%$ male, $60 \%$ ASA III/IV. $57 \%$ had nosocomial infection and $45 \%$ presented generalized peritonitis. Cardiovascular, respiratory and renal complications occurred in $45 \%, 36 \%$ and $9 \%$ of patients respectively.

There were no differences between PIRO score, $\mathrm{MPI}$ and PSS to assess cardiovascular, respiratory and renal complications ( $p$-value $=0.05,0.05$ and 0.39 respectively) (figure $1-3$ ). The cut-off value for cardiovascular, respiratory and renal complications was 25, 24 and 27 for MPI; 10, 10 and 11 for PSS and 6 for PIRO for each one of the complications.

\section{CONCLUSIONS}

PIRO score, PSS and MPI, estimated in patients with IAI admitted to a SICU, could be helpful identifying which patients may develop major complications. Our data suggest that any of the scores analyzed could be appropriate. Nevertheless, cross-sectional studies are required to validate a score that predicts the risk of major postoperative complications.

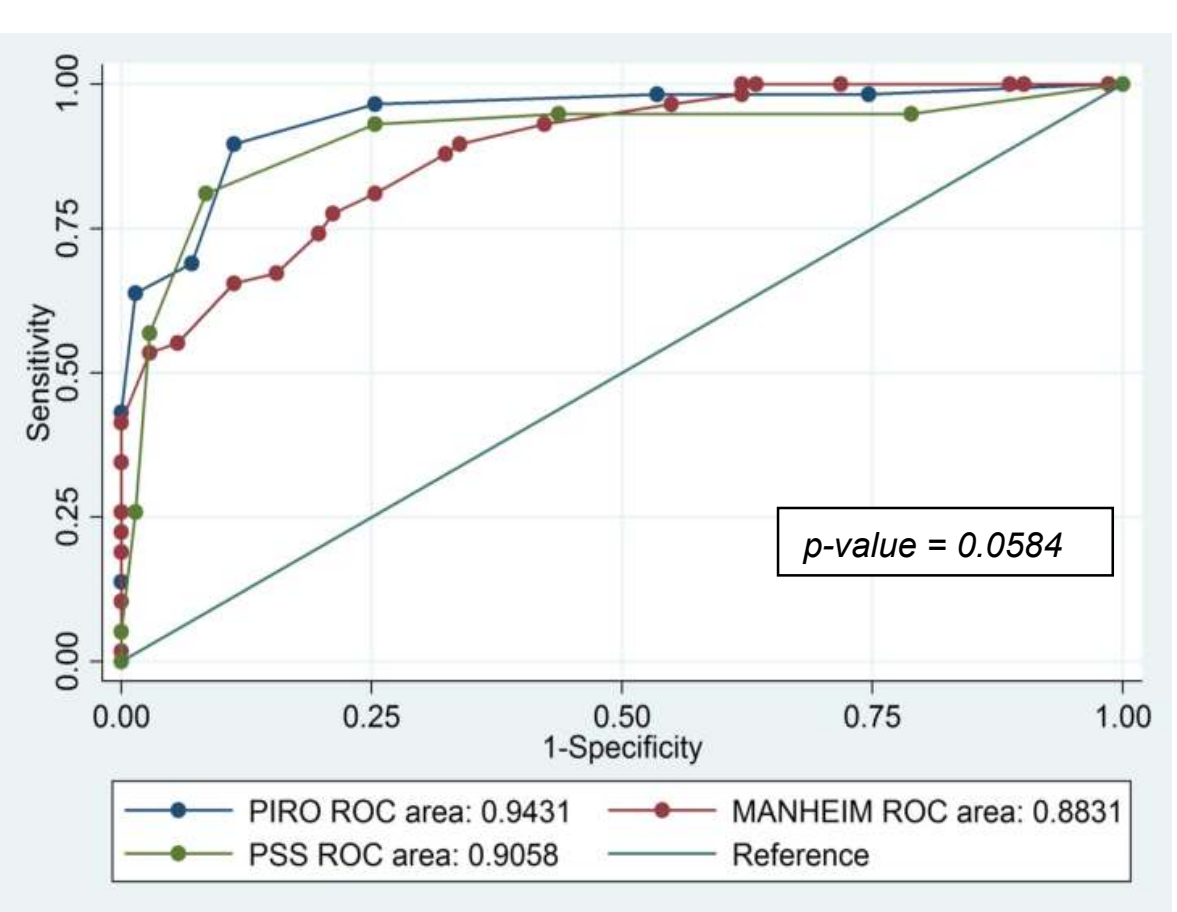

Figure 1. Comparison of ROC curves in predicting risk of cardiovascular complications.

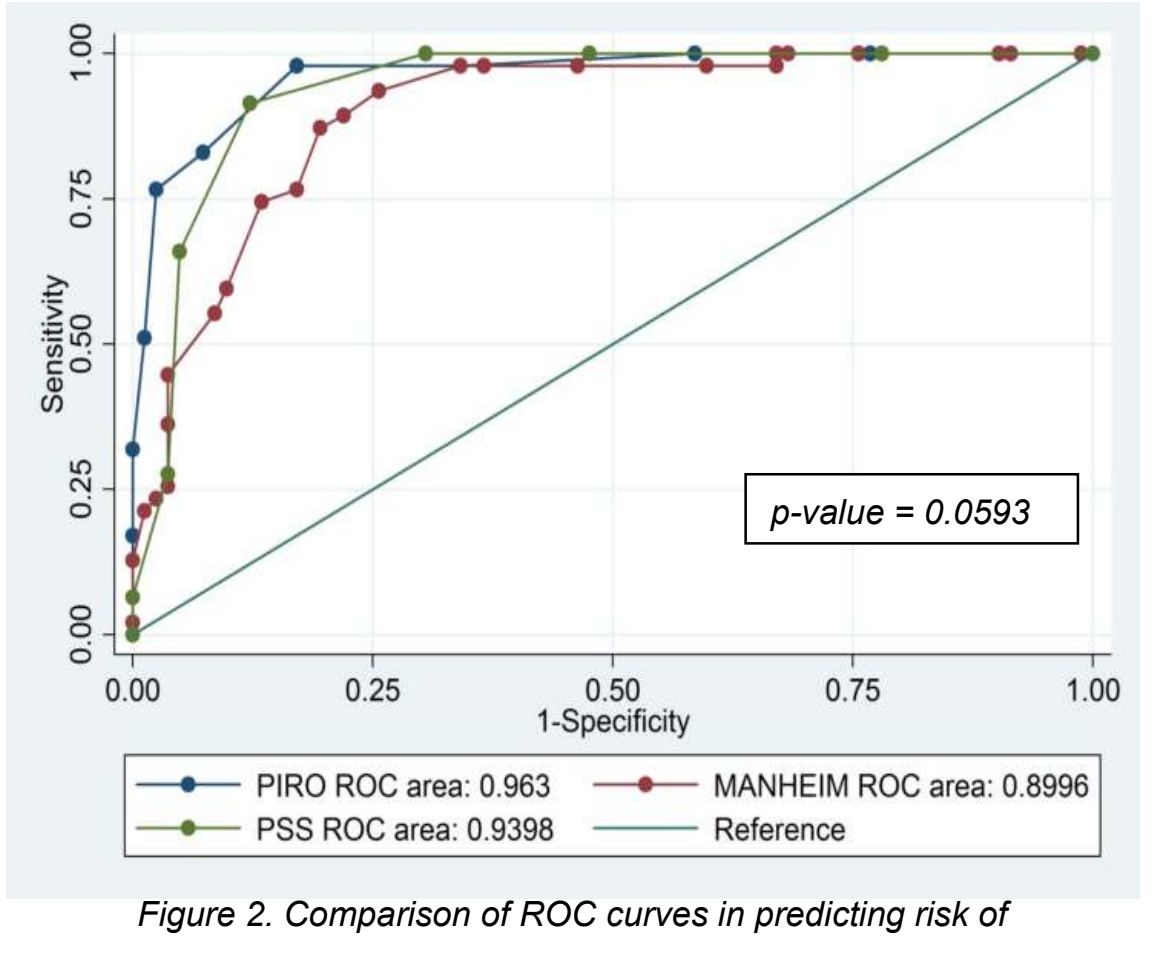

respiratory complications.

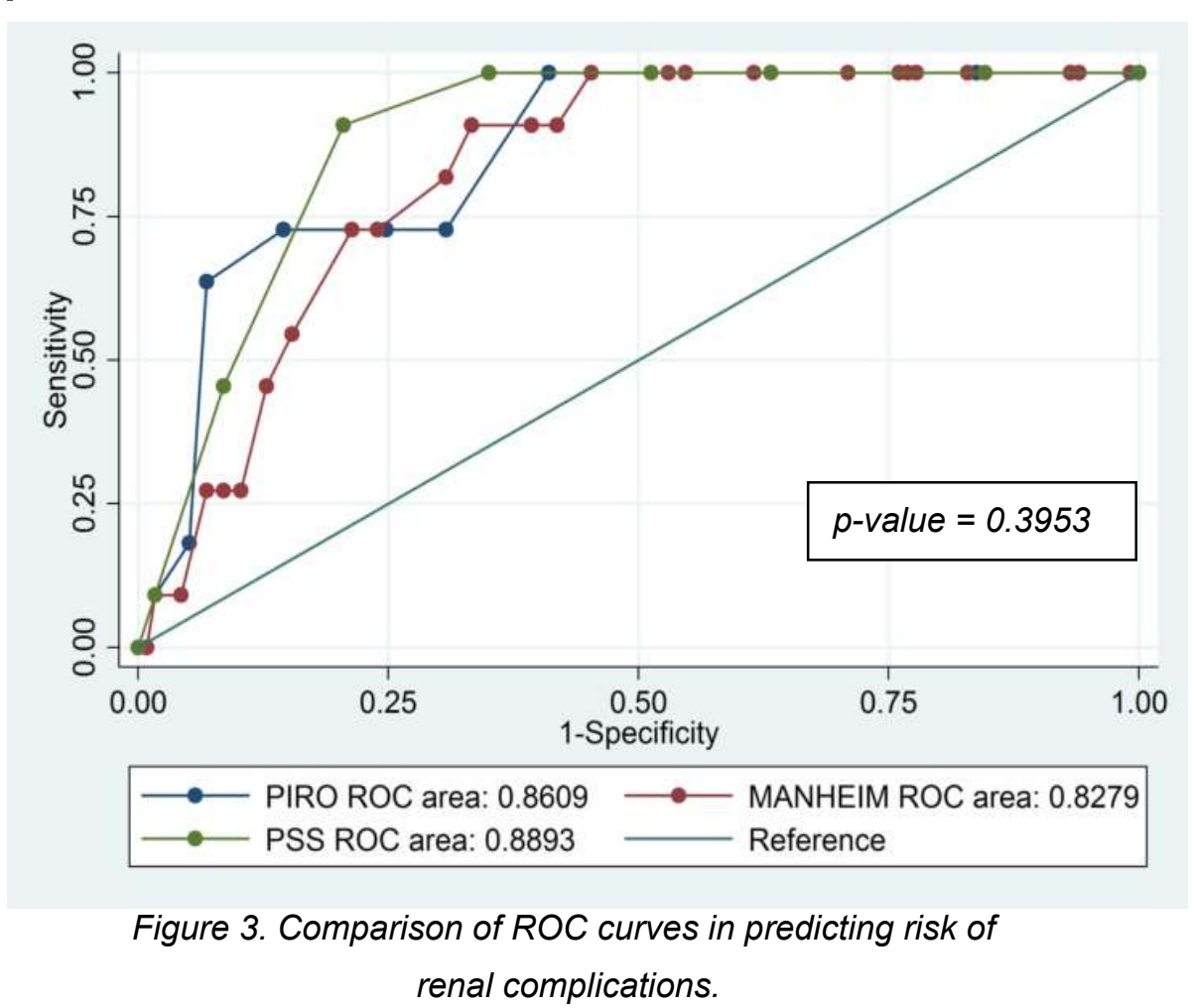

renal complications. 